\title{
Status and implications of neutrino masses: a brief panorama
}

\author{
José W.F. Valle*
}

Instituto de Física Corpuscular (CSIC-UV)

Parc Científic de la Universitat de València

C/ Catedrático José Beltrán, 2

E-46980 Paterna, Valncia, Spain,

* https://www.astroparticles.es/

With the historic discovery of the Higgs boson our picture of particle physics would have been complete were it not for the neutrino sector and cosmology. I briefly discuss the role of neutrino masses and mixing upon gauge coupling unification, electroweak breaking and the flavor sector. Time is ripe for new discoveries such as leptonic CP violation, charged lepton flavor violation and neutrinoless double beta decay. Neutrinos could also play a role in elucidating the nature of dark matter and cosmic inflation.

\section{Introduction}

Neutrinos are the most ubiquitous particles in the universe, over $300 / \mathrm{cm}^{3}$ coming from the Big Bang cross us every second. If cosmological neutrinos were the only ones available probably there would be no neutrino physics, given their incredibly tiny interaction cross sections. Fortunately nature is more generous and stars, such as our Sun, are copious sources of higher energy neutrinos that can be detected say, in gigantic underground detectors like Superkamiokande. Likewise, neutrinos arising from cosmic ray interactions in the upper atmospheric arrive the Earth from 
all directions of the sky. Here too, the agreement between theory and experiments requires the oscillation hypothesis, characterized by a nearly maximal angle $\theta_{23}$, surprisingly at odds from expectations based upon the quark sector.

The resolution of the long-standing discrepancies between theoretical expectation and experimental measurements of solar and atmospheric neutrinos has opened this century with a revolution in particle physics, by providing the first solid evidence for new physics and the need to revise Standard Model of particle physics 1 . The latter assembles the fundamental constituents in three generations of quarks and leptons whose interactions are dictated by the principle of $\mathrm{SU}(3)_{\mathrm{c}} \otimes \mathrm{SU}(2)_{\mathrm{L}} \otimes \mathrm{U}(1)_{\mathrm{Y}}$ gauge invariance. It provides a precise theory of particle interactions, well tested up the highest energies so far explored in particle accelerators. While the photon and the gluon, carriers of electromagnetic and the strong force, are massless, the weak interaction messengers, the $\mathrm{W}$ and the $\mathrm{Z}$ are massive, along with all of the quarks and leptons. The basic theory relies on the principle of gauge invariance and this forbids mass. The simplest way out is the spontaneous electroweak symmetry breaking mechanism, which implies the existence of a physical Higgs boson. Its historic discovery three years ago led many to say that the standard model is now complete. However, the long-standing discrepancies between theoretical expectations and experimental measurements of solar and atmospheric neutrinos requires the existence of neutrino flavor oscillations ${ }^{2}$, and hence the existence of nonzero neutrino masses ${ }^{\sqrt{3}}$. This discovery has triggered a revolution in particle physics, as it provides the first solid evidence for new physics and the need to revise Standard Model. Indeed, particle physics would have been "completed" with the Higgs boson discovery, were it not for the need to account for neutrino oscillations as well as the cosmological puzzles such as dark matter, baryon asymmetry and inflation. In this talk I will give a brief summary of the current landscape of particle physics in view of these issues.

\section{Neutrino mixing and oscillations}

The long-standing discrepancy between theoretical expectation and experimental measurements of solar neutrinos has finally been resolved in favor of the oscillation mechanism, characterized by an angle $\theta_{12}$, substantially larger than its CKM analogue, the Cabbibo angle $\theta_{C}$. Similarly, the agreement between theory predictions and measurements of atmospheric neutrinos at underground experiments, both event yields and angular distributions, indicates the need for neutrino oscilla- 
tions, characterized by a nearly maximal mixing angle $\theta_{23}$, quite different from its quark sector analogue.

Both solar and atmospheric neutrino discrepancies were crucially confirmed by the results of Earth-bound experiments based at reactors and accelerators. For example, the reactor experiment KamLAND pinned down that oscillations is the mechanism underlying the conversion of solar neutrinos and identified the relevant region of oscillation parameters, characterized by a "small" angle $\theta_{12}$, as opposed to oscillations in vacuum. Recent reactor and accelerator experiments have also provided a good measurement of the third lepton flavor mixing parameter $\theta_{13}$, with a first hint of leptonic CP violation just emerging, characterized by a CP phase $\delta$ which promises to open a new era in neutrino physics.

The basic ingredient needed to describe neutrino oscillations is the lepton mixing matrix $K$, which comes from the mismatch between the charged and neutral mass matrices arising after the spontaneous electroweak and lepton number breaking. If neutrinos get mass a la seesaw (see below) then one expects that the heavy neutrino messengers will couple, subdominantly, in the charged current weak interaction leading to a rectangular form ${ }^{3}$ for the matrix $K$.

To analyze the current solar, atmospheric, reactor and accelerator neutrino oscillation data one normally assumes the simplest unitary form for $K$. The two extra physical CP phases present in $K$ are called Majorana phases and are most transparently expressed in terms of the original symmetric parametrization 3 . However they appear only in neutrinoless double beta decay and other lepton number violation processes. Hence they are omitted in neutrino oscillation analyses, for which the symmetric and the PDG presentations coincide.

The summary of the oscillation parameters after the Neutrino 2014 conference are presented in Fig. 1 (more discussion in Lisi's talk). Clearly one has good determinations of all the oscillation parameters except for the leptonic CP phase, which is just making its first appearance in the scene. The squared mass splitting parameters are tiny, without any counterpart in the charged fermion sector. Likewise, the values obtained for the solar and atmospheric angles $\theta_{12}$ and $\theta_{23}$, are much larger than their CKM counterparts. The nonzero value of the reactor angle $\theta_{13}$ opens the door to future leptonic $\mathrm{CP}$ violations studies at the upcoming reactor and accelerator neutrino experiments, such as LBNF-DUNE. The measurement of the leptonic CP-phase using atmospheric neutrinos has been discussed in Smirnov's talk. 

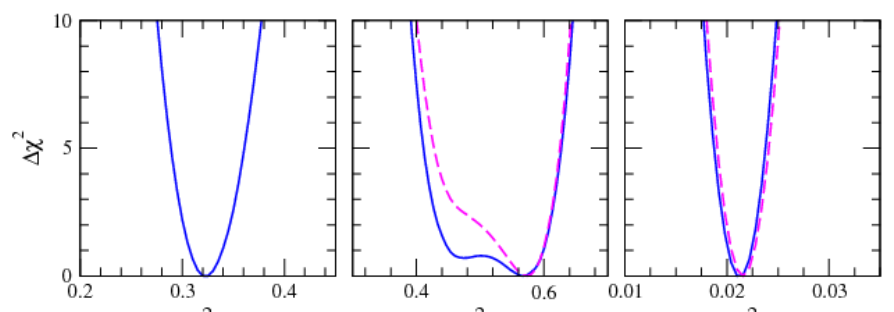

$\sin ^{2} \theta_{12}$

$\sin ^{2} \theta_{23}$ $\sin ^{2} \theta_{13}$

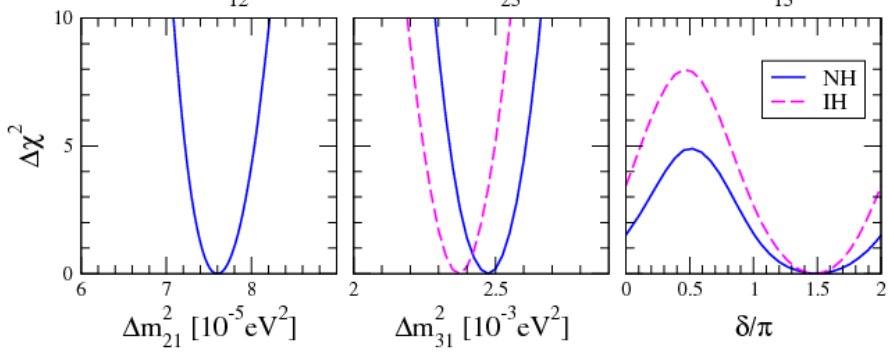

Fig. 1. Global picture of neutrino oscillation parameters after Neutrino-2014, from Ref.2

\section{Effective neutrino mass, seesaw mechanism and unification}

In the standard model neutrinos are massless so we need new physics in order to account for neutrino mixing and oscillations. As noted by Weinberg, one can add non-renormalizable operators, such as the dimension five operator shown in Fig. 2 , that break lepton number and which would account for the small observed neutrino masses. We have no clues as to the characteristic scale, the underlying mechanism or the flavor structure of the relevant operator. If anything, the neutrino oscillation observations indicate a very special pattern of mixing parameters, unlikely to be accidental. Its explanation from first principles, along with the other fermion masses and mixing parameters, constitutes the so-called flavor problem, one of the most stubborn problems in particle physics, and one for which the simplest gauge paradigm falls short at addressing. Here we stress the challenge of reconciling small CKM mixing parameters with large lepton mixing angles within a predictive framework.

The most popular way to induce the operator in Fig. 2 is through the exchange of heavy messengers, as present in $\mathrm{SO}(10)$ Grand unified theories (GUTS). In this case the smallness of neutrino mass is dynamically explained by minimizing the Higgs potential through a simple "1-2-3" vev (vacuum expectation value) seesaw relation of the type

$$
v_{3} v_{1} \sim v_{2}^{2} \text { with the hierarchy } v_{1} \gg v_{2} \gg v_{3} \text {. }
$$




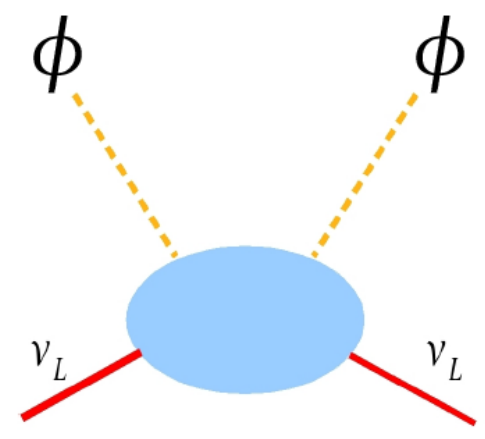

Fig. 2. Weinberg dimension five lepton number violation operator leading to neutrino mass.

The isosinglet vev $v_{1}$ drives the spontaneous breaking of lepton number symmetry and induces also a small but nonzero isotriplet vev $v_{3}$ which generates the $\nu \nu$ entry in the neutrino mass matrix. Since the isodoublet $v_{2}$ fixes the masses of the weak gauge bosons, $W$ and $Z$, one sees that $v_{3} \rightarrow 0$ as $v_{1} \rightarrow \infty$. The most popular messengers are heavy "right-handed" neutrinos (type-I seesaw) and heavy triplet scalar with a small induced vev (type-II seesaw). Although these arise naturally in the framework of $\mathrm{SO}(10)$ GUTS, they may be introduced simply in terms of the $\mathrm{SU}(3)_{\mathrm{c}} \otimes \mathrm{SU}(2)_{\mathrm{L}} \otimes \mathrm{U}(1)_{\mathrm{Y}}$ structure.

\section{New physics: to unify or not to unify?}

Despite the solid evidence for physics beyond-the-Standard Model in the neutrino sector, most theoretical extensions, such as grand unification, have so far been mainly driven by aesthetical principles. Grand unified theories (GUTS) realize one of the most elegant ideas in particle physics. The three observed gauge interactions of the Standard Model which describe electromagnetic, weak, and strong forces merge into a single one at high energies. GUTS bring a rationale to charge quantization and the quantum numbers of the Standard Model. They are thought of as an intermediate step towards the ultimate theory of everything, which would also include gravity. As a generic feature, GUTS break the baryon number symmetry, allowing protons to decay in many ways. To date, all attempts to observe proton decay have failed. Here we stress three attractive features of GUTS:

- Simplest GUTS embed $\mathrm{SU}(3)_{\mathrm{c}} \otimes \mathrm{SU}(2)_{\mathrm{L}} \otimes \mathrm{U}(1)_{\mathrm{Y}}$ in an enlarged simple Lie group, characterized by a single unified gauge coupling constant. 

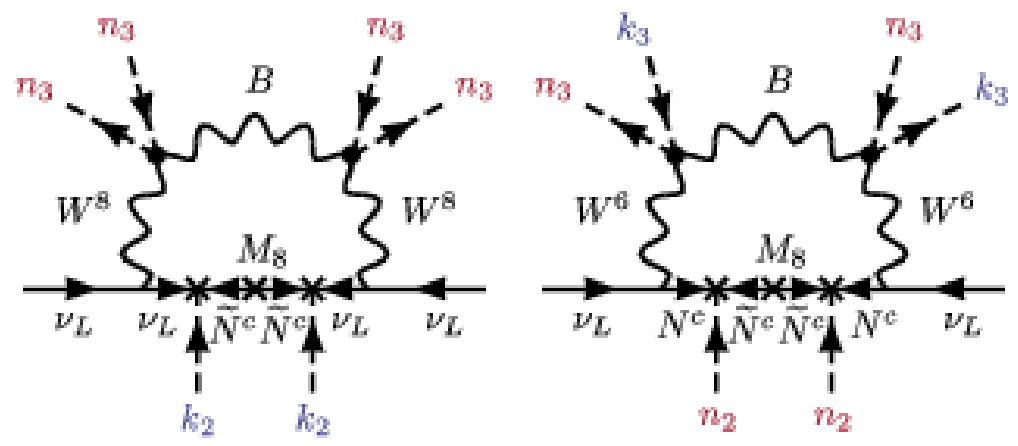

Fig. 3. Two diagrams which contribute to the light neutrinos mass matrix

- GUTS open the door to the possibility of relating quark and lepton masses.

- GUTS like SO(10) require the existence of right-handed neutrinos and the required breaking of B-L implies massive neutrinos.

Here we show how non-unified extended electroweak models with massive neutrinos may unify the gauge couplings as well as relate quark and lepton masses.

\subsection{Neutrino masses without GUTS}

Given that the number and properties of the messengers leading to Fig. 2 are to a large extent arbitrary, one can devise a variety of low-scale realizations of the seesaw paradigm, putting it literally "upside-down". In particular, the seesaw may be naturally realized at low-scale, for example, the inverse and the linear seesaw mechanism. While these can be formulated in a GUT context ${ }^{4}$, this is not necessary at all $\underline{5}$.

An alternative low-scale approach to neutrino masses is to assume that they arise only radiatively, typically as a result of extended symmetry breaking sectors. However, interesting examples have recently been suggested where neutrino masses arise from new gauge interactions, as illustrated in Fig. 3. The crosses denote vev insertions of the relevant scalar multiplets responsible for symmetry breaking in the relevant extended $S U(3)_{C} \otimes S U(3)_{L} \otimes U(1)_{X}$ electroweak setup ${ }^{6}$.

\subsection{Gauge coupling unification without GUTS}

Within the standard $\mathrm{SU}(3)_{\mathrm{c}} \otimes \mathrm{SU}(2)_{\mathrm{L}} \otimes \mathrm{U}(1)_{\mathrm{Y}}$ gauge theory gauge coupling unification constitutes a "near miss". What kind of new physics could make the gauge coupling constants unify "exactly"? The first possibility is having a full-fledged 
Grand Unified Theory, as described above. This, however, entails as phenomenological implication the existence of proton decay, so far unobserved.

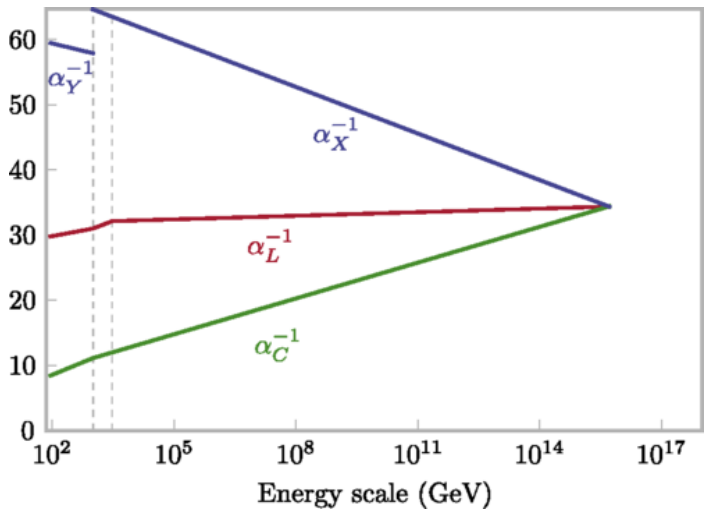

Fig. 4. Gauge coupling unification in $S U(3)_{C} \otimes S U(3)_{L} \otimes U(1)_{X}$ model at $3 \mathrm{TeV}$, from Ref. 6 .

Alternatively, low energy supersymmetry would provide a simple way to account for gauge coupling unification. Such "completion" would however require "sparticles" accessible at the LHC, so far not seen. While we look forward to possible signs of supersymmetry in the next run of the LHC, we note that the physics responsible for gauge coupling unification may be the same inducing small neutrino masses.

A realization of such "GUT-less" unification scenario employs the $S U(3)_{C} \otimes$ $S U(3)_{L} \otimes U(1)_{X}$ electroweak gauge structure, "explaining" why there are three generations from anomaly cancellation ${ }^{7}$. Neutrino masses arise radiatively in the presence of three fermion octets as illustrated in Fig. 4. Altogether, one finds that such "neutrino completion" scheme unifies the gauge couplings thanks to the existence of new states providing neutrino mass. These may lie in the TeV range and hence be accessible to the LHC.

\subsection{Generalized $b-\tau$ unification without GUTS}

Flavor symmetries have been suggested as a way to put order in the "flavor chaos". Here we stress the striking fact that such symmetries have the potential of relating quark and charged lepton masses, in the absence of unification. Indeed, in a class of such $\mathrm{SU}(3)_{\mathrm{c}} \otimes \mathrm{SU}(2)_{\mathrm{L}} \otimes \mathrm{U}(1)_{\mathrm{Y}}$ models one can obtain a canonical mass relation 8

$$
\frac{m_{b}}{\sqrt{m_{d} m_{s}}} \approx \frac{m_{\tau}}{\sqrt{m_{e} m_{\mu}}}
$$


between down-type quark and charged lepton masses. This formula can be understood from the group structure, when there are three vacuum expectation values but only two invariant contractions determining the Yukawa couplings. Note that Eq. 2 provides a successful multi-generation generalized b-tau unification scenario which, moreover, does not require the existence of grand-unification. Note also that it relates mass ratios instead of absolute masses.

\section{Predicting neutrino oscillation parameters}

A remarkable feature, which came as a surprise, is that the smallest of the lepton mixing angles is similar to the largest of the CKM mixing parameters, the Cabibbo angle, while the solar and atmospheric mixing parameters are rather large ${ }^{2}$. One phenomenological approach is to take the reactor angle, similar to the Cabibbo angle, as the universal seed for quark and lepton mixing. Such bi-large schemes point towards Abelian flavor symmetry groups and Frogatt-Nielsen-type schemes ${ }^{910}$. It has been noted however that the observed neutrino mixing angles take very special values, atmospheric mixing being nearly bi-maximal with solar mixing nearly trimaximal. Hence a tri-bimaximal pattern seems reasonable as a starting point $\underline{11}$. Although the full pattern might occur accidentally, it seems that nature is telling its message here: (i) observations seem to suggest some symmetry, and (ii) we must also redefine our strategy in flavor model-building. The challenge is to reconcile the large lepton mixing with the small CKM parameters in a predictive way.

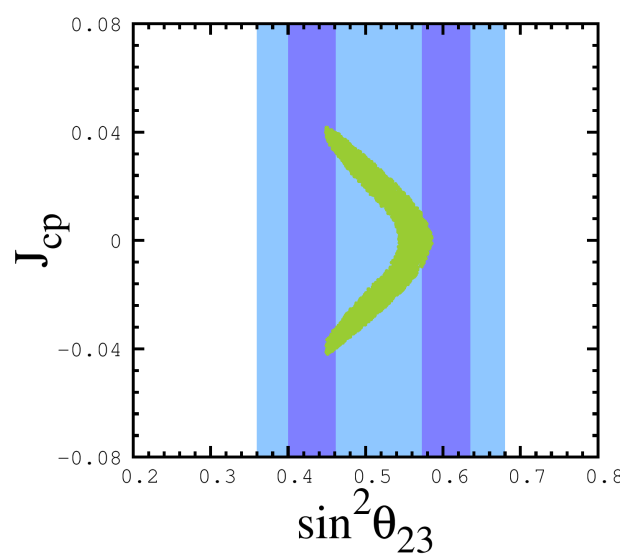

Fig. 5. Correlating $\mathrm{CP}$ violation in neutrino oscillations with the octant of the atmospheric mixing parameter $\theta_{23}$, adapted from Ref.13. 
As a first step one can assign the three lepton families to a three-dimensional irreducible representation of a non-Abelian flavor symmetry group, the smallest one being $A_{4}$. This opens the way to the possibility of predicting the pattern on neutrino oscillation parameters. As simplest zeroth-order predictions one obtains ${ }^{12}$ a maximum atmospheric mixing parameter $\theta_{23}=\pi / 4$ and zero reactor angle $\theta_{13}=$ 0 , with a possible prediction as also for the solar angle, a la tri-bimaximal.

However recent neutrino oscillation data from reactors and accelerators measure a nonzero $\theta_{13}$ value, requiring the early models to be revamped so as to induce a nonzero $\theta_{13}$, without spoiling the other prediction(s). This has been done in a minimal way in Ref. $\frac{13}{13}$, leading to a striking predicted correlation between the magnitude of $\mathrm{CP}$ violation in neutrino oscillations and the octant of the atmospheric mixing parameter $\theta_{23}$ illustrated in Fig. 5 One sees that, at face value, the left octant necessarily violates CP. Time will tell whether this predicted correlations is right. Finally we note that flavor-symmetry-based models often predict the pattern charged lepton flavour violation processes 14 .

\section{Neutrinos and electroweak symmetry breaking}

After the Higgs boson discovery at CERN it is natural to imagine that all symmetries in nature are broken spontaneously. It is also reasonable to imagine that the smallness of neutrino mass is due to the feeble breaking of lepton number, which can be realized in many ways, see below. This requires an extension of the standard model Higgs sector and, if the minimal $\mathrm{SU}(3)_{\mathrm{c}} \otimes \mathrm{SU}(2)_{\mathrm{L}} \otimes \mathrm{U}(1)_{\mathrm{Y}}$ structure is kept, there must be a physical Nambu-Goldstone boson, generically called majoron ${ }^{1}$.

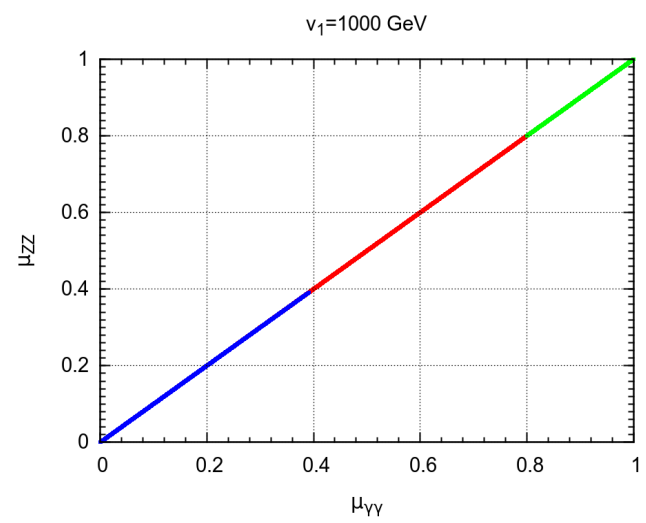

Fig. 6. Correlation between $\mu_{Z Z}$ and $\mu_{\gamma \gamma}$. The points in green pass all constraints, from Ref.15]. 
Although the detailed properties of the majoron in general depend on the model, the existence of new invisible Higgs decays is generically expected, if lepton number violation takes place at or below the weak scale. This is easy to arrange, leading to missing momentum signals at colliders ${ }^{16}$. Given the good agreement of the results from ATLAS and CMS with the standard model Higgs scenario 19 one can place limits on the presence of invisible channels. Within the simplest $\mathrm{SU}(3)_{\mathrm{c}} \otimes \mathrm{SU}(2)_{\mathrm{L}} \otimes \mathrm{U}(1)_{\mathrm{Y}}$ spontaneous low-scale lepton number violation scenario one finds that the current LHC restrictions on the Higgs boson decay branchings can be summarized as in Fig. 6. where the parameters $\mu_{Z Z}$ and $\mu_{\gamma \gamma}$ are "signal-strength" parameters. This restriction still leaves an important chunk of Higgs boson mass and mixing parameters to be explored at the next run of the LHC. Many alternative richer electroweak breaking sectors leading to the double breaking of electroweak and lepton number symmetries can be envisaged.

\section{Neutrinoless Double Beta Decay}

As we saw neutrino oscillations are insensitive to the absolute neutrino mass scale. This can be probed using cosmological data as well as tritium beta decay endpoint studies ${ }^{1}$. A specially interesting complementary approach is the search for neutrinoless double beta decay. While the two-neutrino double beta decay has been experimentally observed in many isotopes, so far we have only experimental lower bounds on the half-lives for the neutrinoless mode 20 . However the latter is expected, on general grounds, to take place at some level, due to the existence of neutrino mass. Using the previous oscillation parameters and leaving the values of the Majorana phases free, one obtains the two broad branches corresponding to the cases of normal and inverted hierarchies indicated in Fig. 7. The horizontal and vertical lines indicate future expected sensitivities. Models based upon flavor symmetries often lead, as phenomenological predictions, to correlations between the neutrino oscillation parameters. In a large class of such models these translate as lower bounds for the effective mass parameter $\left|m_{e e}\right|$ characterizing $0 \nu \beta \beta$ decay even for the normal mass ordering. This is seen as the two dark sub-regions in Fig. 7. Many other models leading to a lower bound on the $0 \nu \beta \beta$ decay rate can be constructed 21 .

In gauge theories $0 \nu \beta \beta$ can be induced in many ways other than the neutrino exchange or "mass mechanism". For example, there can be short-range mechanisms involving the exchange of heavy particles such as present in left-right or super- 


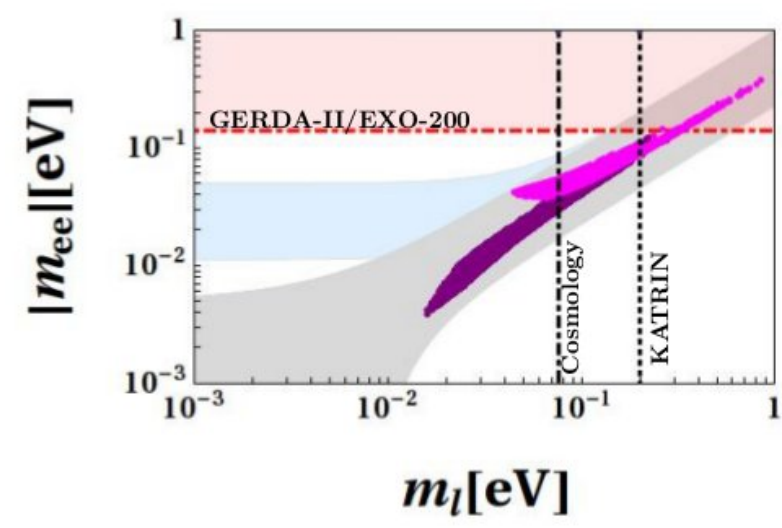

Fig. 7. Neutrinoless double beta decay effective amplitude parameter versus the lightest neutrino mass, in a generic model versus a flavor-symmetry-based model, from Ref. 8 .

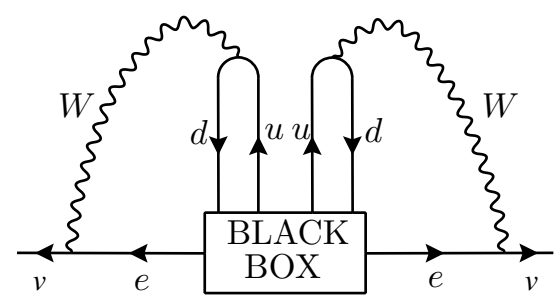

Fig. 8. Neutrinoless double beta decay implies Majorana neutrinos, from Ref. ${ }^{[22}$.

symmetric extensions of the standard model ${ }^{23224}$. The significance of neutrinoless double beta decay comes from the fact that, whatever the mechanism responsible for $0 \nu \beta \beta$ in a gauge theory one can always "dress" the corresponding amplitude with $\mathrm{W}$ bosons, showing that a Majorana neutrino mass is necessarily induced ${ }^{22}$, as illustrated in Fig. 8 . This theorem holds under very general assumptions, as shown by Lindner and collaborators ${ }^{25}$.

\section{Neutrinos and cosmology}

Neutrinos affect the cosmic microwave background (CMB) and large scale structure in the Universe, playing a key role in the synthesis of light elements, which takes place when the universe is about a few minutes old. The feeble interaction of neutrinos allows us to use them as cosmic probes, down to epochs far earlier than we can probe with optical telescopes. The current cosmological puzzles associated with the baryon number of the universe, inflation and dark matter are probably 
associated to epochs earlier than the electroweak phase transition at $\sim 10^{-12}$ sec. It is not inconceivable that (some of) these puzzles may have a common origin with the physics driving neutrino masses ${ }^{1}$, as schematized in Fig. 9.

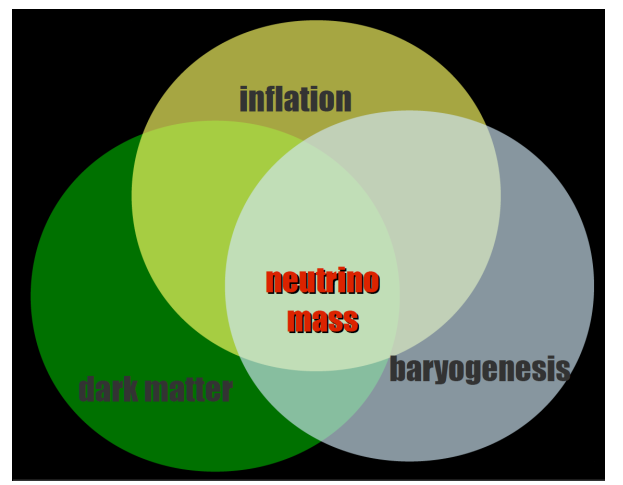

Fig. 9. Cosmological puzzles possibly associated to the same physics that drives neutrino mass.

Here we focus on the possibility that neutrino masses arise from spontaneous breaking of ungauged lepton number. The associated Nambu-Golstone boson may acquire mass from lepton number violation by quantum gravity effects at the Planck scale 2627 . If its mass lies in the keV range, the weakly interacting majoron can play the role of dark matter particle, providing both the required relic density as well as the scale for galaxy formation 28 . Since the majoron couples to neutrinos proportionally to their tiny mass, it is expected to be very long-lived, stable on cosmological scales $\underline{29}$. Though model-dependent, its coupling to the charged leptons is expected to be very weak so the majorons produced during the phase transition may never be in thermal equilibrium during the history of the universe. Alternatively they could be in thermal equilibrium only for some period. The lifetime and mass of the latedecaying dark matter majoron consistent with the cosmic microwave background observations can be determined 3132 . Moreover, as a pseudoscalar, like the $\pi^{0}$, the dark matter majoron will have a (sub-dominant) decay to two photons, leading to a characteristic mono-energetic X-ray emission line $\mathrm{e}^{32 / 33}$. These features fit nicely in models where neutrino masses arise from a type-II seesaw mechanism.

A recent twist along these lines was the proposal that inflation and dark matter have a common origin (similar idea was suggested by Smoot in arXiv:1405.2776 [astro-ph]), with the inflaton identified to the real part of the complex singlet containing the majoron and breaking lepton number through its vev $\underline{30}$. The resulting 


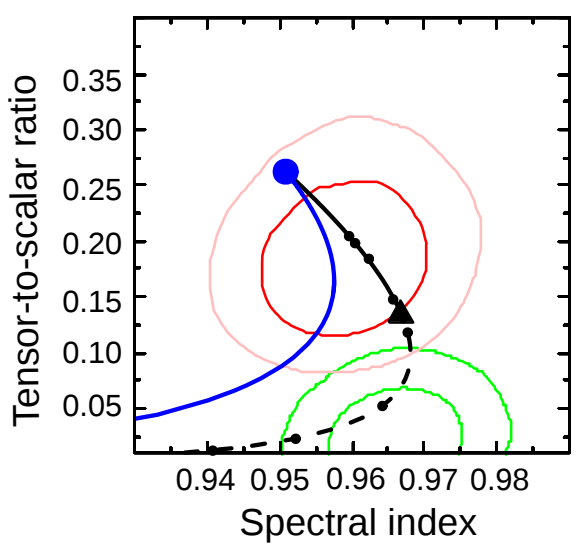

Fig. 10. Cosmological predictions of seesaw inflation and majoron dark matter model of Ref. 30 .

inflationary scenario is consistent with the recent CMB observations, including the B-mode observation by the BICEP2 experiment re-analized jointly with the Planck data, as illustrated in Fig. 10. The upper (red) contours correspond to the BICEP2 results, while the lower ones (green) follow from the new analysis released jointly with PLANCK ${ }^{34}$. The lines correspond to 68 and 95\% CL contours. Further restrictions on the majoron dark matter scenario should follow from structure formation considerations.

Work supported by MINECO grants FPA2014-58183-P, Multidark CSD200900064, and the PROMETEOII/2014/084 grant from Generalitat Valenciana.

\section{References}

1. Extensive discussion in J. W. F. Valle and J. C. Romao, Neutrinos in high energy and astroparticle physics. Wiley-VCH, Berlin, 1st edition ed., 2015.

2. D. Forero, M. Tortola, and J.W.F. Valle, "Neutrino oscillations refitted,"

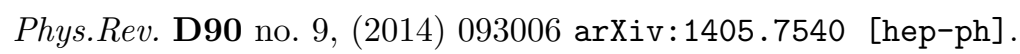

3. J. Schechter and J.W.F. Valle, "Neutrino Masses in SU(2) x U(1) Theories," Phys.Rev. D22 (1980) 2227.

4. M. Malinsky, J. Romao, and J.W.F. Valle, "Novel supersymmetric $\mathrm{SO}(10)$ seesaw mechanism," Phys.Rev.Lett. 95 (2005) 161801

5. S. M. Boucenna, S. Morisi, and J. W. F. Valle, "The low-scale approach to neutrino masses," Adv. High Energy Phys. 2014, 831598 (2014)

6. S. M. Boucenna et. al., "Small neutrino masses and gauge coupling unification" Phys. Rev. D 91 (2015) 031702. 
7. M. Singer, J. Valle, and J. Schechter, "Canonical Neutral Current Predictions From the Weak Electromagnetic Gauge Group SU(3) X u(1)," Phys.Rev. D22 (1980) 738

8. S. Morisi et. al., "Relating quarks and leptons without grand-unification," Phys. Rev. D 84 (2011) 036003; S. Morisi et. al., "Quark-Lepton Mass Relation and CKM mixing in an A4 Extension of the Minimal Supersymmetric Standard Model," Phys. Rev. D 88 (2013) 036001; S. F. King et, al., "Quark-Lepton Mass Relation in a Realistic $A_{4}$ Extension of the Standard Model," Phys. Lett. B 724 (2013) 68; C. Bonilla et. al., "Relating quarks and leptons with the $T_{7}$ flavour group," Phys.Lett. B742 (2015) 99

9. S. Boucenna, S. Morisi, M. Tortola, and J. Valle, "Bi-large neutrino mixing and the Cabibbo angle," Phys.Rev. D86 (2012) 051301

10. G.-J. Ding, S. Morisi, and J. Valle, "Bilarge neutrino mixing and Abelian flavor symmetry," Phys.Rev. D87 no. 5, (2013) 053013

11. P. Harrison, D. Perkins, and W. Scott, "Tri-bimaximal mixing and the neutrino oscillation data," Phys.Lett. B530 (2002) 167,

12. K. Babu, E. Ma, and J. Valle, "Underlying A(4) symmetry for the neutrino mass matrix and the quark mixing matrix," Phys.Lett. B552 (2003) 207-213, arXiv:hep-ph/0206292 [hep-ph].

13. S. Morisi, D. Forero, J. C. Romao, and J. W. F. Valle, "Neutrino mixing with revamped A4 flavour symmetry," Phys.Rev. D88 (2013) 016003, arXiv:1305.6774 [hep-ph].

14. S. Morisi and J. W. F. Valle, "Neutrino masses and mixing: a flavour symmetry roadmap," Fortsch.Phys. 61 (2013) 466-492,

15. C. Bonilla, J. W. F. Valle, and J. C. Romo, "Neutrino mass and invisible Higgs decays at the LHC," arXiv:1502.01649 [hep-ph]

16. A. S. Joshipura and J. W. F. Valle, "Invisible Higgs decays and neutrino physics," Nucl. Phys. B397 (1993) 105-122.

17. F. de Campos et al., "Searching for Invisibly Decaying Higgs Bosons at LEP II," Phys. Rev. D55 (1997) 1316-1325.

18. DELPHI collaboration Collaboration, J. Abdallah et al., "Searches for invisibly decaying Higgs bosons with the DELPHI detector at LEP," Eur. Phys. J. C32 (2004) 475-492, hep-ex/0401022.

19. ATLAS, CMS Collaboration, G. Aad et al., "Combined Measurement of the Higgs Boson Mass in $p p$ Collisions at $\sqrt{s}=7$ and $8 \mathrm{TeV}$ with the ATLAS and 
CMS Experiments," arXiv:1503.07589 [hep-ex].

20. A. Barabash, "75 years of double beta decay: yesterday, today and

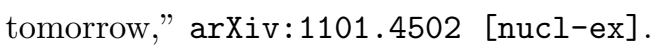

21. L. Dorame, D. Meloni, S. Morisi, E. Peinado, and J. Valle, "Constraining Neutrinoless Double Beta Decay," Nucl.Phys. B861 (2012) 259-270

22. J. Schechter and J. Valle, "Neutrinoless Double beta Decay in $\mathrm{SU}(2)$ x U(1) Theories," Phys.Rev. D25 (1982) 2951.

23. F. Bonnet, M. Hirsch, T. Ota, and W. Winter, "Systematic decomposition of the neutrinoless double beta decay operator," JHEP 1303 (2013) 055

24. S. Das, F. Deppisch, O. Kittel, and J. Valle, "Heavy Neutrinos and Lepton Flavour Violation in Left-Right Symmetric Models at the LHC," Phys.Rev. D86 (2012) 055006, arXiv:1206.0256 [hep-ph].

25. M. Duerr, M. Lindner, and A. Merle, "On the Quantitative Impact of the Schechter-Valle Theorem," JHEP 1106 (2011) 091

26. S. B. Giddings and A. Strominger, "Loss of Incoherence and Determination of Coupling Constants in Quantum Gravity," Nucl.Phys. B307 (1988) 854.

27. T. Banks and N. Seiberg, "Symmetries and Strings in Field Theory and Gravity," Phys.Rev. D83 (2011) 084019, arXiv:1011.5120 [hep-th].

28. V. Berezinsky and J. Valle, "The KeV majoron as a dark matter particle,"

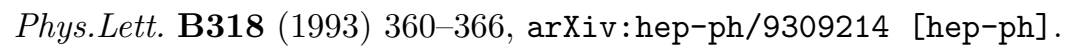

29. J. Schechter and J. Valle, "Neutrino Decay and Spontaneous Violation of Lepton Number," Phys.Rev. D25 (1982) 774.

30. S. Boucenna, S. Morisi, Q. Shafi, and J. Valle, "Inflation and majoron dark matter in the seesaw mechanism," Phys.Rev. D90 (2014) 055023

31. M. Lattanzi and J. Valle, "Decaying warm dark matter and neutrino masses," \begin{tabular}{|l|l|l|}
\hline Phys.Rev.Lett. 99 (2007) 121301, arXiv:0705.2406 [astro-ph] \\
\hline
\end{tabular}

32. M. Lattanzi, S. Riemer-Srensen, M. Tortola, and J. Valle, "Constraints on majoron dark matter from cosmic microwave background and astrophysical observations," Nucl.Instrum.Meth. A742 (2014) 154-157.

33. M. Lattanzi, S. Riemer-Sorensen, M. Tortola, and J. W. F. Valle, "Updated CMB and $\mathrm{x}$ - and $\gamma$-ray constraints on Majoron dark matter," Phys.Rev. D88 (2013) 063528, arXiv:1303.4685 [astro-ph.HE]

34. BICEP2, Planck Collaboration, P. Ade et al., "Joint Analysis of BICEP2/Keckarray and Planck Data," Phys.Rev.Lett. 114 (2015) 101301 